\title{
ECONOMÍA INDÍGENA EN LA COMUNIDAD DE WASAKÍN, MUNICIPIO DE ROSITA, RAAN
}

\author{
Pío Alejandro García Izaguirre ${ }^{[1]}$ \\ Saida de los Ángeles Argüello Mendieta ${ }^{[2]}$
}

\section{Resumen}

Se presenta el estudio de caso: Economía Indígena de Wasakín, municipio de Rosita, Región Autónoma del Atlántico Norte (RAAN), a 480 Km al noreste de Managua, Nicaragua. La población está constituida por seis familias tuahkas, el resto están enlazados con la etnia tuahka y mísquitu. La población aproximada es de 2,100 habitantes, conformados por 185 familias, prevalece la identidad tuahka y mískitu. Se asentaron aproximadamente en 1733 en el río Bambana, a $13.5 \mathrm{Km}$. de la ciudad de Rosita en el reinado de Eduardo I (1728-1762).

Se identificaron el modelo de economía, las actividades productivas, cultura, la percepción del pueblo en referencia al modelo de su economía. La investigación fue cualitativa con métodos de la Antropología Cultural. Los habitantes viven de la agricultura rudimentaria, madera, ganadería, artesanía de bambú y servicios de medicina tradicional, comercio, caza, pesca y recolección. Existe pobreza y el Estado ha fomentado tradicionalmente el paternalismo con donaciones realizadas por cada gobierno.

Palabras clave: Economía indígena, renglones de economía, bienestar económico, percepción y alternativa económica.

\section{Summary}

We present a case study on Indigenous economy in Wasakín, municipality of Rosita, North Atlantic Autonomous Region (RAAN), located at $480 \mathrm{~km}$ northeast of Managua, Nicaragua. The population is constituted by six Tuahka families, the rest are linked with the Tuahka-Miskitu ethnic group. The population is approximately 2,100 people, made up of 185 families; the tuahka and miskitu identity prevails. These families settled approximately in 1733 in the Bambana River, at $13.5 \mathrm{~km}$ from the city of Rosita, during the reign of Edward I (1728-1762).

The economic model was identified, as well as the productive activities, culture, and the perception of the people in reference to its economic model. The research was qualitative and linked with methods of cultural anthropology. The people live

\footnotetext{
[1] Máster en Antropología Social. Coordinador IEPA, URACCAN-Las Minas. palejandrogi@yahoo.es

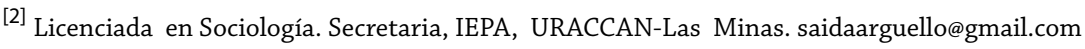


from the rudimentary agriculture, wood, cattle-raising, bamboo crafts, traditional medicine services, trade, hunting, fishing and gathering. There is poverty and the State has traditionally fostered paternalism with donations made by each government.

Keywords: Indigenous economy, rows of economy, economic wellbeing, economic perception, economic alternative.

\section{Introducción}

Esta investigación presenta características particulares de la economía del pueblo mayangna tuahka de la comunidad Wasakín, municipio de Rosita, Región Autónoma Atlántico Norte (RAAN). Es importante destacar que de este estudio ya se ha presentado la primera parte: Economía indígena de Sikilta, de población panamahka del municipio de Siuna, tríangulo minero. Lo cual permite establecer comparaciones entre ambas economías de dos pueblos distintos.

El artículo se caracteriza por ser un estudio de caso, de carácter descriptivo propio de la Antropología Cultural. En principio, consiste en identificar y analizar la cultura económica de la comunidad. Las preguntas fueron formuladas con el fin de conocer la percepción de la población y la propuesta comunitaria para mejorar la economía. La economía de los indígenas está basada en la agricultura, ganadería, comercio de madera, artesanía, caza, pesca recolección, comercio, venta de servicios de salud tradicional.

En el marco teórico, se identifican modelos de economía de algunos países de Mesoamérica. En el caso de México, el modelo de economía pre-capitalista de subsistencia; en Guatemala los mayas, la economía tiene un perfil capitalista primitivo vinculado al comercio nacional; en Honduras los tawahkas, son agricultores que trabajan para la subsistencia y el autoconsumo.

En la segunda parte, se abordan las características de la economía, se describen los renglones de la agricultura, ganadería, caza, pesca, recolección, artesanía, venta de madera, leña y los servicios de medicina tradicional por curanderos y parteras. Finalmente, las autoridades y las personas de la comunidad presentan propuestas para obtener apoyo en las áreas agropecuaria, social, ambiental, infraestructura para salir de la pobreza y de la dependencia del gobierno del Estado.

Finalmente, las personas fundadoras y líderes de la comunidad presentan propuestas para obtener apoyo en el área agropecuaria, y lo social para mejorar las condiciones sociales de la población y salir de la pobreza. 


\section{Revisión de literatura}

En los países de Mesoamérica mejorar la economía de los pueblos indígenas es una demanda presente, dominante en nuestro medio.

Tania Calva (2000), en México, se refirió al modelo de economía indígena y lo describe como pre capitalista de subsistencia. [...]. Los rasgos culturales a partir de los cuales se define la situación actual de los pueblos indígenas de Mesoamérica son vida sedentaria y cultivos de maíz, frijol y calabaza (...). El comercio es también una actividad primordial llevada a cabo por hombres y sobre todo mujeres indígenas que venden sus productos en los numerosos mercados del país. Existe tambien una pequeña pero significativa élite cultural indígena principalmente urbana, que encabeza el trabajo desde muchas organizaciones indígenas.

En Honduras los Tawahkas, [...] se dedican al renglón económico de la agricultura, incluyendo las mujeres y los niños. Cultivan plátanos, malanga, guineo (banano), camote, yuca, amarga y dulce, caña de azúcar, ayote, frijoles y café. La agricultura es de subsistencia, y practican la caza, pesca, recolección, en pequeña escala para el consumo familiar.

Las transformaciones de la economía indígena a monetaria a raíz de la introducción de empresas madereras, huleras, mineras, pesqueras, en los centros urbanos: Entre 1860 y 1894 se acentuó la tradicional diferencia entre la economía indígena, orientada casi exclusivamente a la subsistencia; y la economía en manos extranjeras, cuyo fin exclusivo era el mercado mundial (Romero Vargas, German (1996).

Entre 1905 y 1979 el desarrollo de la región del Atlántico estuvo en manos de compañías extranjeras, las poblaciones locales continuaron desarrollando sus actividades tradicionales. Las necesidades de mano de obra de las compañías y la escasa población local tuvieron consecuencias imprevistas en la vida social y cultural. [...] Recordemos a este respecto que dos modos de vida distintos se habían contrapuesto en la región.

Antes de 1894, por un lado, el modo de vida tradicional de una economía de subsistencia, practicado por los indígenas como por los criollos (pp.164). La economía de las comunidades indígenas del tríangulo minero es agrícola. (...) En Siuna la comunidad de Sikilta, los cultivos más frecuentes que siembran son arroz, frijol, maíz, caña, yuca, plátano, malanga, quequisque, ayote, batata, pijibay, marañón, pera, jocote, banano (Ruiz Calderón, 2006).

Peralta Justo \& Indalecio Samuel (1997), la misma está determinada por actividades tradicionales, que les permite la sobrevivencia mediante la venta de animales 


\section{CULTURA INDÍGENA Y AFRODESCENDIENTE}

domésticos, agricultura, pesca, carne de monte, animales vivos, oro, leña y madera, botes y artesanía, y los excedentes los venden en el mercado de Siuna.

Gotz Friherr (2003), en el estudio sobre la economía indígena en Bonanza dijo:

La economía de los mayangnas se basa en los renglones de caza y pesca, hoy ha disminuido tanto los peces y los animales silvestres... [...] La caza es esporádica de pequeños animales, tales como guatusa, guardatinaja, iguana, tortugas y pescados pequeños. Además crían animales domésticos: gallinas, algunos patos, gallinas de Guinea, pavos, cerdos negros pequeños y algunas vacas flacas de mala raza que con dificultad producen 2 litros de leche.

El PNUD describe aspectos de la economía de la subzona agro silvícola indígena del norte de Nicaragua, por ejemplo: En Bilwi Puerto Cabezas las comunidades indígenas, mayangnas, miskitus, garífunas, ramas y afrodescendientes están más orientadas al autoconsumo que al mercado, aunque en algunos casos como el de las comunidades del Llano Sur, venden en Bilwi hasta el 30\% de su producción. Igual ocurre en Waspam, cuyas comunidades muestran mayor nivel de integración al mercado local de Bilwi y con algunas localidades de Honduras.

Gómez, Suarez (2003), informa sobre la economía de los tawahkas de Honduras: La estructura económica de los tawahkas se caracteriza por ser eminentemente agraria. Los cultivos más frecuentes son frijoles, arroz, yuca, mismos que siembran en las riberas opuestas al pueblo. La economía de subsistencia es la actividad productiva principal de los tawahkas, aunque desde mediados del siglo pasado estos han participado en la economía nacional global. Su participación siempre ha sido a través de la participación de mano de obra a las compañías nacionales e internacionales que entraban en la mosquitia bajo concesiones entregadas por el gobierno (p.147).

García Izaguirre, Pio Alejandro (2006), en investigación efectuada en Wasakín encontró que los conflictos son de carácter económico por venta de tierras indígenas por mestizos y a la vez la economía se caracteriza por ser de subsistencia.

\section{Materiales y métodos}

Se realizó investigación documental, entrevistas en profundidad semi-estructuradas, la observación participante con guía de acuerdo a sus variables, con informantes claves, hombres, mujeres y autoridades de la comunidad. Para la selección de los informantes se recurrió al método de la bola de nieve que consiste en seleccionar por 
recomendación de otro del mismo círculo. En el trabajo de campo todas las variables tuvieron respuesta.

\section{Resultados y discusión}

\section{Actividades económicas por prioridad}

Las actividades económicas de la comunidad por prioridad son: la artesanía, agricultura y, maderas: en segundo lugar la caza, pesca, recolección y en tercer lugar la ganadería. Estos rubros tienen la siguiente dinámica.

\section{Artesanía}

En la comunidad existe un colectivo con un taller de artesanía en el que construyen mobiliario con la caña de bambú. Los productos los venden en la comunidad a precios favorables pero los intermediarios que distribuyen en el tríangulo minero venden a precios carísimos.

En el caso de los artesanos pagan por el bambú que utilizan y así generan ingreso para la comunidad. El 5\% es el canon de interés que debe de pagarse a la comunidad, para mejorar la estructura de la escuela. Pero esto no ocurre, porque los beneficios de la artesanía son para las personas y familiares que trabajan la artesanía.

Para la elaboración de objetos de bambú los artesanos compran el material a $C \$ 1.00$ córdoba cada caña de bambú con el que construyen en el barrio Cuyusbinphine y venden sillas a $C \$ 300.00$ o $C \$ 600.00$, mesas de $C \$ 200.00$ a $C \$ 500.00$, camas a $C \$ 1,000.00$, cunas de $C \$ 400.00$ a $C \$ 1,200.00$ y juegos de sala a $C \$ 1,500.00$. Los precios dependen del tamaño pero en la comunidad estos objetos son baratos no así en Siuna; un juego de muebles completo vale $C \$ 3,000.00$ córdobas.

\section{Agricultura}

La población en su mayoría de dedica a la agricultura y siembran principalmente arroz, frijoles y maíz, muy pocos tubérculos y musáceas. La producción es para consumo familiar y el poco excedente lo venden en la comunidad o en el mercado municipal. La producción no es suficiente para la subsistencia familiar ni para comprar artículos como alimentos, ropa, medicinas y perfumes.

La agricultura es la principal fuente de ingresos, a pesar que se caracteriza por ser incipiente por impulsar actividades mediadas por la cultura que no les permite mejorarla. 
Las áreas que siembran no van más allá de $25 \mathrm{~V}^{2}$ hasta $4 \mathrm{Mz}^{2}$ como promedio. El siguiente cuadro refleja las características específicas de la actividad agrícola:

Cuadro No.1. La agricultura en Wasakín 2010

\begin{tabular}{|c|c|c|c|}
\hline Cultivos & Área cultivada & $\begin{array}{l}\text { Producción x } \\
\text { familias }\end{array}$ & $\begin{array}{l}\text { Producción qq } \mathbf{x} \\
\text { comunidad }\end{array}$ \\
\hline Arroz & 1/2, 1,3,2 Manzanas(Mz) & $\begin{array}{l}60,70,74 \text { quintales } \\
\text { (qq). }\end{array}$ & $800 \mathrm{qq}$ \\
\hline Maíz & $25 \mathrm{~V} 2,1 / 2,1,2,3 \mathrm{Mz}$ & $30,50,63,99$ & $\begin{array}{l}\text { Venden } 34 \text { y } 36 \text { para } \\
\text { consumo. }\end{array}$ \\
\hline Frijoles & $252,1 / 2,1,2 \mathrm{Mz}$ & $20 \mathrm{qq}$ & $\begin{array}{l}100 q q / 12 \text { qq consumo, } \\
8 \text { venden. }\end{array}$ \\
\hline Plátano & $3.21 \mathrm{Mz}$ & $\begin{array}{l}\text { 32,100 plátanos } \\
(1,287 \text { racimos }) .\end{array}$ & $\begin{array}{l}\text { Toda la comunidad } \\
1,119(87 \%) \text { consumo y, } \\
167 \text { venden }(13 \%)\end{array}$ \\
\hline Banano & $\begin{array}{l}1 \mathrm{Mz}, 70,100,200,300 \\
\text { matas. }\end{array}$ & & $\begin{array}{l}\text { Variedad Felipita, } \\
\text { jocote. }\end{array}$ \\
\hline Yuca & $1,2,4,1 / 2 \mathrm{Mz}$ & $\begin{array}{l}57 \text { qq. ( } 1 \mathrm{Mz} \text { el que } \\
\text { siembra). }\end{array}$ & $\begin{array}{l}\text { Producen menos que } \\
\text { el consumo. / Venden } \\
\text { toda la producción. }\end{array}$ \\
\hline Malanga & $1 / 2 m z$ & & 1 productor \\
\hline Quequisque & $\begin{array}{l}25 V_{2} \\
1 / 4 \mathrm{~V} 2\end{array}$ & $\begin{array}{l}100 \mathrm{lbs} \\
20 \mathrm{qq}\end{array}$ & $\begin{array}{l}1 \text { productor } \\
1 \text { productor }\end{array}$ \\
\hline
\end{tabular}

Fuente: Información estructurada por el autor.

\section{Herramientas para la agricultura}

Las herramientas agrícolas que utilizan los indígenas son igual a las que utilizan los mestizos. La propiedad de herramientas agrícolas se manifiesta en las siguientes cantidades: 2 machetes por productor, 2 limas, 1 hachas, 1 piocha, algunos poseen 1 espeque, 2 productores poseen bombas para fumigación, y 27 sacos para empaque por familia productora. El concepto de arado lo desconocen, porque dijeron que tienen 7 , pero el investigador en 10 años de observación no ha identificado ninguno. Esto mismo ocurre con la cultura mestiza que es una minoría que posee esta herramienta agrícola

\section{Comercio de recursos naturales}

Como parte de las actividades económicas venden plantas de maderas preciosas y semipreciosas para reforestación: caoba, cedro real, cedro macho, maría, laurel y pino. Los precios de cada planta es de C\$: 40.00 córdobas. 


\section{Maderas}

No toda la poblacion es productora de madera y los que se dedican a su extracción reflejan mejores condiciones de vida. Se observa que cuentan con refrigerador, plantas de gasolina, pequeños negocios con artículos en tres líneas: alimentación, ropa, medicinas y perfumes. Las casas lucen ser las mejores de concreto, madera nueva y pintadas.

El segundo bloque de prioridades se ubica dentro de actividades ancestrales, caza, pesca y recolección. Estas actividades son milenarias y los recursos naturales se han agotado y, no generan ingresos económicos, pero en la realidad actual siguen siendo importantes aun en el período de la modernidad igual a las comunidades de poblacion mestiza.

La explotación de madera es el segundo rubro de la economía en la comunidad, con la diferencia de que este negocio está en manos de unos 40 madereros, cuyas utilidades son para los que ejercen la actividad.

Las especies de madera explotadas más frecuentemente se reflejan en las siguientes variedades en un año. Por ejemplo, los entrevistados expresaron que en un año un maderero cortó 59 caobas, 68 cedros reales, 116 cedros machos, 143 marías, 5 laureles, 8 areno y 7 comenegro. Estos recursos naturales los extraen los madereros mediante autorización del síndico y la asamblea comunal y, los planes de manejo los aprueba el MAGFOR en Rosita.

\section{Madera en rollo}

En la comunidad hay un alto tráfico de madera para comercialización, de 12 entrevistados $8(67 \%)$, cortan madera para comercio, estos tienen permisos que oscilan entre $6 \mathrm{Mts} 3-600 \mathrm{Mts} 3$, en total 1,701.5 Mts 3 (721,436.0o pies tablares).

\section{Leña}

De 12 familias 9 (75\%) cortan leña para el comercio, unas 109,00o unidades de leña, con promedio en un año de 12,111.11 unidades por familia. La unidad la venden $C \$$ 1.00 córdoba en la comunidad. La venta de leña es un producto que siempre han llevado al mercado.

\section{Tecnología de explotación de madera}

Para la extracción de madera lo que más utilizan los nativos son las moto sierras, unas 30 familias posee esta máquina y, además cada familia tiene entre 1 lima y 2 limas, 
dos machetes, 1 hacha, mazo y sierra tradicional de mano. De cada 12 familias dos poseen un bote o dos como medio de transporte.

\section{La caza, pesca y recolección}

\section{La caza}

Siguen siendo cazadores de animales silvestres, más bien para la subsistencia diaria en la que participan adultos. Pareciera ser que la cacería aún sigue siendo prometedora por las cifras que indican que los cazadores devastaron cientos de animales silvestres. En el 2010 los entrevistados dijeron que cazaron: 2,012 guatusas, 1,560 guardiolas, 1,035 cusucos, 1,736 iguanas, 807 venados, 161 saínos, 6o loras, 1 ardilla, o chancho de monte, o lapas. Esta información contrasta con la observación realizada por el investigador a partir de los años 2002- 2010, y refiere haber observado la cacería de 1 venado, 1 saíno, 4 iguanas en un período de ocho años.

La tecnología aplicada en la cacería continua siendo tradicional, cada familia tiene dos perros que lucen esqueléticos, acompañan a cazadores que poseen a nivel comunitario 10 rifles calibre No. 22, 6 escopetas, 6 lanzas y 11 arcos con flechas.

\section{La Pesca}

Históricamente, la pesca ha tenido dos utilidades importantes para los indígenas de esta comunidad, para consumo familiar y venta. En el año 2010, 11 pescadores reportan que capturaron 3,537 guapotes, 4,810 sabaletes, 3,570 mojarras, 5,600 barbudos, 2,450 robalos, 4,203 iguanas y 10 tortugas.

Pero en la misma comunidad, en observación sistemática verificada por el investigador en 8 años, un pescador tenía en venta con su esposa no más que unas 30 libras de guapotes (mojarras), 2 tortugas (de más o menos 3 libras) o pez -barbudos de tamaños pequeños, dejándose los diminutos para el consumo familiar-. Todo hace indicar que la pesca ya no es rentable para venta ni para consumo familiar, menos para considerar que es un rubro que genera utilidades debido a que fue arrasada en el Siglo XX.

La tecnología pesquera sigue siendo la tradicional, en cada familia hay anzuelos, arpones, máscaras y pistoletas. Es más común observar algunos cuantos barbudos extraídos de lagunas, y pintos que los niños pescan por la mañana de aproximadamente 1, 2, onzas de peso y tamaños diminutos. 
Gotz Friherr (2003), en el estudio sobre la economía indígena en Bonanza dijo:

La economía de los mayangnas se basa en los renglones de caza y pesca, hoy han disminuido tanto los peces y los animales silvestres... [...] La caza es esporádica de pequeños animales, tales como guatusa, guardatinaja, iguana, tortugas y pescados pequeños. Además crían animales domésticos gallinas, algunos patos, gallinas de guinea, pavos, cerdos negros pequeños y algunas vacas flacas de mala raza que con dificultad dan 2 litros de leche.

\section{La recolección}

La recolección es una actividad ancestral en los pueblos originarios. En la comunidad recolectan plantas medicinales, frutas silvestres, semillas, miel de abejas, hojas para techos, bejucos, considerados de gran importancia para alimentación, medicina, y materiales de construcción para viviendas.

\section{Ganadería}

El tercer rubro en importancia es la ganadería. Existen unos 21 productores que poseen fincas con iniciativas ganaderas, aproximadamente de 50 hectáreas de tierra cada una o más, y tienen la perspectiva de aumentar el área, de apropiarse de más tierras, ya que son libres para trabajarla.

Estas familias trabajan para construir un sistema productivo de carácter individual y privado diferente al sistema de economía comunal. Estas iniciativas incipientes pero modernas para ellos tienen finalidades claves: producir para beneficio de una sola familia y formar una barrera de contención mediante la ubicación de fincas para evitar el posicionamiento de nuevos colonos mestizos en el territorio y salir de la pobreza, obtener dinero, casa, ganadería, vehículos a igual que los mestizos.

La comunidad requiere de la gestión para la asistencia técnica para 21 pequeños ganaderos poseedores de parcelas de tierra en carácter privado dentro del territorio comunal donde practican la ganadería de forma incipiente, con la existencia alrededor de 134 cabezas de ganado bovino, unos cuantos equinos cerdos y gallinas. Los problemas que tiene la ganadería son tórsalos, neumonía y sarna.

La ganadería es embrionaria y crían pocas reses, unos cuantos caballos, pelibuey, principalmente cerdos y gallinas, las cabezas de ganado mayor y menor las venden en el mercado comunitario o en el mercado más cercano de Rosita. 


\section{CULTURA INDÍGENA Y AFRODESCENDIENTE}

La ganadería y tecnología aplicada es incipiente, tienen unas 3 jeringas de vacunación y 15 bombas de fumigación con más aplicación en la agricultura en limpieza de cultivos. No hay dinero para comprar medicamentos, cuatro familias tienen recursos y pueden comprar medicamentos para su ganado y otros no tienen finca.

\section{Medicina convencional}

La comunidad posee un centro de salud deteriorado y asistido por un enfermero, pero en lo general está desprovisto de medicina y en algunos casos permanece cerrado. La comunidad posee saberes que le han permitido sobrevivir en todos los períodos de la historia, con sus recursos humanos y naturales para enfrentar enfermedades comunes y temibles propias del trópico húmedo.

El servicio de salud que ofrece el MINSA es malo, no hay medicamentos para cubrir las necesidades de toda la comunidad, el personal médico lo compone sólo una persona, hay atención por un enfermero pero no hay medicinas. Se necesita construir el centro de salud, pintarlo, no existen fondos, por lo que no pueden hacer nada. Otros valoran que la atención de la salud está entre regular porque no hay suficientes medicamentos y terceros consideran buena porque facilitan medicamentos a enfermos.

Otros recursos de los que se vale la población, son medicamentos convencionales que se encuentran de venta en la comunidad: acetaminofén, panadol, enteroguanil, ibuprofeno, diclofenac, aspirina, primaquina, dolovitalgia, mata dolor, dolofin, zepol, enteromebac, alka-seltzer, espasmofin. Las enfermedades más comunes que padecen son: dolor sólo diarrea, vómito, dolor de huesos, dolor de espaldas, calentura, dolor de muela, gripe, catarro entre otras.

\section{Curanderos}

En la comunidad existen unos seis curanderos como promedio. Cada uno atiende a 32 pacientes al año con una consulta de $C \$ 144.00$ córdobas por persona. Pero hay un curandero en la comunidad que cobra $C \$ 3,000.00$ córdobas por tratar casos de enfermedades que la medicina convencional occidental no puede curar. Dicen que saben curar el cáncer. El precio para curar depende de la enfermedad que se padece.

\section{Parteras}

Hay nueve parteras, cada una atiende a muchas mujeres en los partos en el año. De acuerdo con la cultura el precio para atender a una mujer de parto cuesta $\$ 130.00$ córdobas y, si es mujer la que nace cuesta $C \$ 63.00$ córdobas, algunas veces lo hacen gratis. Consideran más importante el nacimiento de un niño con respecto al nacimiento de una niña, lo que manifiesta la discriminación de la mujer en esta cultura. 


\section{Transacciones económicas}

Las transacciones económicas de la poblacion de Wasakín las realizan en la comunidad, en el mercado del municipio del Rosita y Puerto Cabezas. Las ventas normalmente son trimestrales, semestrales o al fin del año cuando es período de cosecha de granos básicos. Otros productos tales como tubérculos y musáceas los venden en el mercado local de Rosita, sea cada cada 15 días o mensual.

En general lo que venden es principalmente arroz, frijoles, banano, plátano, pijibay, leña, madera, carne de güilla, algunos cuantos pescados, venado y productos artesanales. Para tener idea del volumen de productos que venden se logran contabilizar 10 racimos de banano, 10 de plátano, 10 de pijibay, y unas 12 libras de güilla.

\section{Beneficios a la población}

Al comercializar madera lo que beneficia a la comunidad son los salarios de trabajo y, el $25 \%$ de interés que le corresponde según la Ley 445 de impuestos que pagan al Estado por venta de madera, permisos que otorgan a madereros, el dinero se ocupa para mejorar la comunidad, casas, andenes, caminos, (carriles), compra de artículos de uso personal.

\section{Comercio}

En la comunidad existen 15 diminutos negocios, en donde venden a la población 44 artículos que corresponden a cuatro rubros, alimentación, utensilios de cocina, cosméticos y ropa:

- Alimentación: arroz, frijoles, azúcar, café, harina, aceite, sal, galletas, gaseosas, sopa maggi, maruchan, confites, granos básicos, bombón.

- Utensilios de cocina: panas plásticas, porras, sillas plásticas, platos, baldes, vasos, fósforos, encendedor, baterías, foco, candela,

- Cosméticos: perfumes, desodorante, jabón, pasta dental, cepillo, espejos, cuadros.

- Vestuario: zapatos, pantalones, camisas, sandalias, mosquiteros, cobijas, papel higiénico, toallas sanitarias.

\section{La cultura y desarrollo}

Según las personas comunitarias, en la cultura mantienen siempre las costumbres y creencias, el idioma prevalece heredado de generación en generación de padres a hijos, así como las comidas y bebidas. Pero esta no contribuye al desarrollo económico porque no tienen suficiente semilla para sembrar para salir de la costumbre, no poseen conocimientos técnicos agrícolas por lo que la producción es baja, generan 
poco dinero, siembran para comer, no hay comercio, no controlan el dinero, no guardan semilla, siembran los mismos cultivos, casi no les gusta trabajar al igual no piensan en el futuro.

\section{Modelo de economía}

El modelo de economía de Wasakín se caracteriza por ser de autoconsumo, en la que desarrollan labores agrícolas en parcelas de tierra por familia, comunal organizadas por parentesco familiar y cooperativas. El mismo pueblo valora la economía como primitiva de subsistencia, al estilo campesino mestizo, con tendencias hacia una economía inicial de mercado por trabajar en parcelas individuales, cuya producción corresponde a la familia que trabajo la parcela.

\section{La producción y dinámica de distribución}

La dinámica de distribución y consumo de los productos a nivel comunitario se basa en la producción por parcela, a cada quien le asignan un pedacito de tierra destinada para sembrar y de allí dan de comer a la familia. La producción que obtiene cada productor es para consumo propio. No existe distribución comunitaria, esa fue una actividad del pasado histórico.

\section{Distribución del tiempo destinado a las actividades productivas}

Cada quien de manera individual distribuye y hace lo que quiere con su tiempo. Realizan actividades de acuerdo al trabajo que hay para darle de comer a la familia. Las actividades agrícolas las realizan en primeras y postreras, pero siembran más en el período de postrera.

\section{Características de la economía en las comunidades mestizas}

La cultura mestiza posee más dinero y ahorran para comprar, cuentan con mejores condiciones, cosechan más, poseen más ganado, tienen su propia tierra con títulos, tienen acceso a préstamos, mejores conocimientos en agricultura, ganadería, en todo son más avanzados, poseen mejores oportunidades, más disponibilidad en el trabajo, piensan en el futuro. "La economía de los mestizos es otra cosa, el mestizo trabaja”(Emilio Fenly Johnny, Wasakín 20 marzo 2010).

Las formas de contratación son de forma oral y los salarios comunes son $C \$ 100.00$, $C \$ 120.00, C \$ 150.00$ al día e incluye la alimentación en los tres tiempos. 


\section{Características de la economía indígena}

Los indígenas crían animales domésticos, promueven la ganadería, artesanía de bambú, trabajan la tierra, comercializan madera, la agricultura, realizan el comercio, la caza, pesca, y recolección. "Los indigenas son distintos porque no llegan a hacer lo que hacen los mestizos, son más avanzados, tienen más conocimientos en la agricultura, comercio y ganadería" (Emilio Fenly Johnny, Wasakín, 20/3/10).

El tipo de relación laboral parte de la dinámica de la actividad económica que se da en Wasakín es el parcelero. Los pequeños productores trabajan en parcelas de tierra. "Una parcela es una porción pequeña de terreno que suele considerarse como sobrante de otra mayor que ha sido comprada adjudicada o expropiada". En el caso de Wasakín son parcelas desmembradas del territorio comunal asignadas por las autoridades de la comunidad para que trabajen la tierra.

Romero Vargas, Germán (1996: p.164) explica las transformaciones de la economía indígena a monetaria a raíz de la introducción de empresas madereras, huleras, mineras, pesqueras, en los centros urbanos:

Entre 1905 y 1979 el desarrollo de la región del Atlántico estuvo en manos de compañías extranjeras. Las poblaciones locales continuaron desarrollando sus actividades tradicionales. Las necesidades de mano de obra de las compañías y la escasa población local tuvieron consecuencias imprevistas en la vida social y cultural. [...] Recordemos que dos géneros de vida distintos se habían contrapuesto en la región antes de 1894 . Por un lado, género de vida tradicional de una economía de subsistencia, practicado por los indígenas como por los criollos.

\section{Semejanzas}

Trabajan la tierra, son agricultores, comercializan madera, crían animales domésticos y sobre todo promueven la ganadería.

\section{Género y actividades que contribuyen a la economía familiar}

Toda la familia trabaja para la sobrevivencia cada quien con su rol, en actividades domésticas y en el area rural, tales son:

Los niños: Juegan, estudian en la escuela, bañan, limpian patio, lavan ropa, ayudan a sus padres en el campo, jalan agua, algunas veces lustran, venden pan o aguacates, buscan leña, pilan arroz y pescan. 
Las niñas: juegan estudian, lavan ropa, se bañan en el río, ayudan a la mamá en la casa, jalan agua, lavan traste, limpian la casa, el patio, cocinan.

Las mujeres: cuidan a los niños y ancianos, lavan, cocinan, limpian la casa, hacen pan para vender, cuidan animales, planchan ropa, ayudan algunas veces al marido en el campo, imparten clases.

Los hombres jóvenes: trabajan la tierra en el campo, pescan, cazan, recolectan, comercializan madera, rajan y llevan leña a la casa, algunos elaboran artesanía y limpian el patio de la vivienda.

Los ancianos: Dan consejos a los jóvenes, median conflictos, ayudan a tomar decisiones, van a la iglesia a orar, cuidan la casa, gestionan para que lleguen proyectos a la comunidad, cuidan los bosques, trabajan en el campo en la agricultura, algunos son artesanos.

\section{Tipo de energía que utilizan}

El tipo de energía utilizado hasta el 2,010 fue foco, candela, candil, palo de pino seco, el MINSA utiliza panel solar. "En la comunidad hay 5 plantas (Misión Morava), el resto pertenecen a madereros, usan leña, candil, pino, ese es mi candil." (Emilio Fenly Johnny, Wasakín, 23 marzo 2010). A partir del 2011 poseen energía las 24 horas del interconectado nacional.

\section{Nutrición}

\section{Lo que contiene la dieta alimenticia diaria es suficiente}

Arroz, frijoles, banano, plátano, cuajada, a veces carne, pescado, huevo, pan, leche, fresco, hacen falta más cosas que no tienen por falta de dinero. A veces cuando la cosecha es buena hay comida.

Hacen falta verduras pero no tienen dinero para mejorar la dieta ni para comprar y sembrar verduras, ni para comprar leche, cuajadas y verduras para que ayude a mejorar la dieta,

Sobre el mismo tema otros líderes opinaron sobre la dieta alimenticia. "Sí, es suficiente porque así son nuestras costumbres" (Arnulfo Taylor, Wasakín, 13 abril 2010). No necesitamos tener mayor conocimiento de cómo sembrar verduras para comer e incluirlas en nuestra dieta. "Cuando no hay cosecha. La dieta es maíz, arroz, frijoles. El pescado, animales silvestres lo consumen muy poco. (Emilio Fenly Johnny, Wasakín 20 marzo 2010). 


\section{Sobre el modelo de la economía de la comunidad}

Con respecto al modelo económico todos expresaron no estar conformes debido a las siguientes causales:

La población no está conforme con el modelo cultural de economía indígena. Necesitan apoyo en semillas de otros cultivos aparte de granos básicos para sembrar más, generar más ingreso y capacitación en organización y técnicas agrícolas. No se puede desarrollar la comercialización. Cuando las cosechas hay alimentación y se compra ropa, medicinas, cuando no hay se aguanta, aunque no se tiene pero aguantan Hacen falta muchas cosas para ser una comunidad desarrollada en infraestructura, casas, andenes, caminos.

No pueden desarrollar la comercialización y necesitan capacitación para una mejor organización. Un comunitario agricultor dijo: "Cuando hay producción se compran otros artículos, cuando no hay se aguanta, aunque no tiene pero aguanta”. La cultura no les ha permitido el desarrollo familiar. La economía es estática, no avanza, no satisface a la población.

\section{Asalariados de Wasakín}

Los salarios tienen diferentes niveles si es que pagan a un trabajador agrícola. Un día de un trabajador es de $C \$ 100.00$, el salario mensual pueden ser variados $C \$ 3,000.00$, $C \$ 1,000.00, C \$ 1,500.00$, sin alimentación, $C \$ 600.00$ y anual según sean los salarios mensuales.

Existen otras modalidades de pago de un mestizo a un indígena, por ejemplo la limpieza de potreros. Los mestizos por un año de trabajo regalan un ternero a un indígena. Ningún indígena le trabaja a un indígena de forma asalariada. Ningún mestizo le trabaja a indígenas.

\section{Propuestas para promover un nuevo modelo socioeconómico}

La gente comunitaria proponen que para mejorar la economía al estilo de la cultura mestiza, requieren apoyo en proyectos en las siguientes áreas: agricultura, medioambiente, capacitaciones, ganadería, educación, salud, agua y saneamiento e infraestructura.

\section{Conclusiones}

Las prácticas económicas tradicionales no contribuyen a mejorar la economía indígena porque los excedentes de los productos agropecuarios cultivados son insuficientes para el comercio y salir de la pobreza. La produccion comunitaria es utilizada para la subsistencia familiar. 
La economía de la comunidad está basada en la agricultura, ganadería, artesanía, cortes de madera, servicio de salud tradicional, caza, pesca y recolección de productos de la naturaleza la que no contribuye para que las familias salgan de la pobreza.

\section{Recomendaciones}

\section{Capacitaciones para la formulación y gestión de proyectos}

- La poblacion de Wasakín solicita apoyo al Estado del municipio de Rosita, región, nacional y a Organismos no Gubernamentales, para el desarrollo socioeconómico de acuerdo a la cosmovisión indígena. Destacan la importancia de la promoción de la economía indígena para fortalecer la identidad, de acuerdo a sus tradiciones e intereses propios y retomar componentes propuestos por líderes comunitarios y retomar ejemplos de la economia del pueblo mestizo para mejoría de la misma.

- Construir un granero para almacenamiento de semillas, granos básicos e implementar los cultivos de cacao. Así mismo, promover los cultivos de plátanos, tomates y quequisque, aplicando técnicas agrícolas actualizadas.

- Instalación de un trillo para sacar la cascarilla al arroz para 25 productores organizados en cooperativa de arroceros, vender servicio de trillado y promover la autosostenibilidad económica.

- En medioambiente: Reforestación con plantas de pino y árboles latifoliados cedro y caoba.

- En corte y confección: Financiamiento a 10 mujeres en liderazgo, comercialización y mediación de conflictos.

- En ganadería: Apoyo a la crianza de ganado mayor y menor, aves de corral con infraestructura básica.

- En educación: Mejorar la asistencia técnica y material en educación primaria, secundaria, becas para los hijos, construir una escuela en Walangwas (5) aulas y Tubalaya (2).

- En salud: Restauración del centro de salud en el centro de la población de Wasakín y, construcción de otro en Tubalaya, abastecido de medicamentos.

- Agua y saneamiento: Construcción de dos pozos artesanales, en Tubalaya y Walpatara.

- Infraestructura: Construcción de tres mil metros de andenes y, un parque para recreación de la niñez de la comunidad.

- Es importante destacar que los proyectos a diseñarse en agricultura y otros rubros socios culturales deben ser de acuerdo a sus prácticas culturales y la cosmovisión de la cultura. 


\section{Lista de referencias}

Calva, Tania (2000). Pueblos Indígenas y Desarrollo. Los Programas de las Agencias Internacionales de Cooperación para el Desarrollo y los Pueblos Indígenas: el caso de Mesoamérica. Editor Universidad del Valle de Guatemala.pp.49.

Gomez Suarez, Aguda (2003). Movilización Política Indígena en las Selvas Latinoamericanas. Los Tawahkas de la Mosquitia centroamericana. Editorial

García Izaguirre, Pio Alejandro (2006). Conflictos Territoriales en la Comunidad de Wasakin Municipio de Rosita Región Autónoma del Atlántico Norte (RAAN). Bilwi, Noviembre 2006. p. 86.

Gotz Friherr (2003). Mayangna Apuntes sobre la historia de los indígenas Sumu en Centroamérica; contribuciones a la etnología centroamericana [Managua: Fundación Vidal], 2003.: il; byn. - (Colección Cultural de Centro América; Serie Etnología $\mathrm{Na}$ 1) $668 \mathrm{p}$.

Programa de las Naciones Unidas para el Desarrollo (PNUD) (2005). Las Regiones Autónomas de la Costa Caribe de Nicaragua Informe de Desarrollo Humano 2005 ¿Nicaragua asume su diversidad?-1a..Managua: PNUD, 2005.P.365.

Peralta \& Indalecio Samuel (1996). Sikilta y situación actual de los Mayangnas Balna del ULI Was/Comp. Justo Peralta, Samuel Indalecio, MARENA, The Nature Conservancy, USAID. Managua v.73p.: il.72 p.

Romero Vargas, German (1996). Historia de la Costa Atlántica, Managua 1996, CIDCA -UCA. Colección Autonomía. ed. Octubre 1996.p.164

Ruiz Calderón, Angélica Leonor (2006). Economía y Relaciones Interétnicas Comunidad Indígena Mayangna de Sikilta. Siuna RAAN. URACCAN Siuna recinto las minas. $86 \mathrm{p}$.

http:/www.ucm.es/info/socio1/prietokp.html(Consultado11 agosto 2009, en línea).

http://www.iwgia.org/sw30458.asp (Consultado 11 agosto 2009 en línea). 